
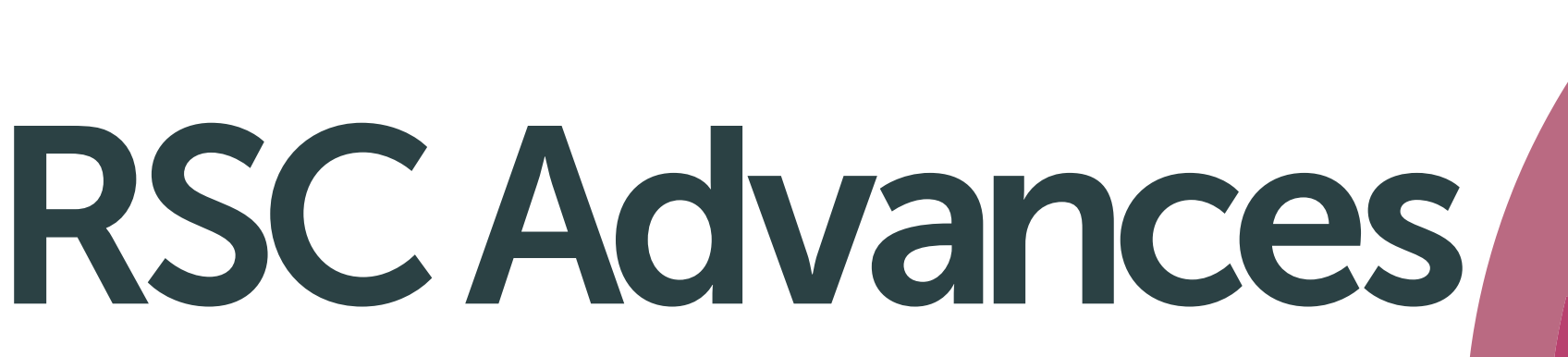

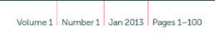

\section{RSC Advances}

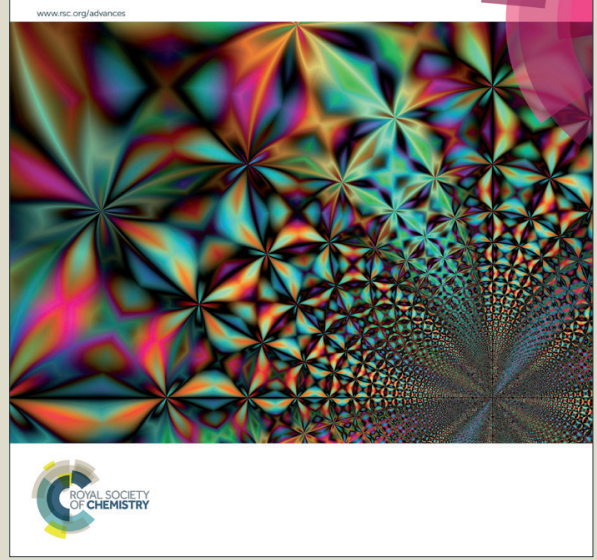

This is an Accepted Manuscript, which has been through the Royal Society of Chemistry peer review process and has been accepted for publication.

Accepted Manuscripts are published online shortly after acceptance, before technical editing, formatting and proof reading. Using this free service, authors can make their results available to the community, in citable form, before we publish the edited article. This Accepted Manuscript will be replaced by the edited, formatted and paginated article as soon as this is available.

You can find more information about Accepted Manuscripts in the Information for Authors.

Please note that technical editing may introduce minor changes to the text and/or graphics, which may alter content. The journal's standard Terms \& Conditions and the Ethical guidelines still apply. In no event shall the Royal Society of Chemistry be held responsible for any errors or omissions in this Accepted Manuscript or any consequences arising from the use of any information it contains. 


\title{
Chitin hybrid material reinforced with graphene oxide nanosheets: Chemical and mechanical characterisation
}

\author{
Joaquín Antonio González, ${ }^{\mathrm{a}}$ María Florencia Mazzobre, ${ }^{\mathrm{b}}$ María Emilia Villanueva, ${ }^{\mathrm{a}}$ Luis Eduardo Díaz \\ and Guillermo Javier Copello*a
}

\author{
Received (in XXX, XXX) Xth XXXXXXXXX 200X, Accepted Xth XXXXXXXXX 200X \\ First published on the web Xth $X X X X X X X X X 200 X$ \\ DOI: $10.1039 / b 00000000 x$
}

Chitin hybrid materials reinforced with graphene oxide nanosheets (nGO) have been prepared. Chitin:nGO ratio ranged from proportions were chitin is the main component to ones where nGO exceed those of chitin. SEM and TEM images showed that high nGO proportions may turn into nanosheets association. FTIR, ${ }^{13} \mathrm{C}$ solid-state NMR and DSC analysis showed that the interaction among the components would not involve the formation of new molecular bondings. nGO was shown to act as a filler that induces structural rearrangements in chitin which lead to new hydrogen bondings among the chains. Mechanical stability proved to be higher when nGO content in the hybrid is similar to or higher than that of chitin. Rheological behaviour showed to be more solid-like with the increment of nGO content. The nGO did not interfere with lysozyme activity over chitin chains indicating that these materials would be biodegradable.

\section{Introduction}

Chitin is the second most abundant biopolymer found in nature after cellulose and can be found in fungi, the exoskeleton of insects and the shell of crustaceans, including shrimp and crab, as well as other invertebrates, such as marine sponges. ${ }^{1,2}$ It is commercially available as a by-product from the food industry with little processing and, therefore, is a low-cost biopolymer. ${ }^{3,4}$ It is a polysaccharide with a structure consisting predominantly of unbranched chains of $\beta$ - $(1 \rightarrow 4)-2$-acetoamido-2-deoxy-D-glucose. In the past decade it has sparked interest as a building block for materials to be applied in a wide range of fields such as medicine, biotechnology, biosorption and food industry. 5 This interest takes root in its biocompatibility, biodegradability, low toxicity and capability of forming mechanical stable materials that can be used for protein sorption, water remediation, food wrapping, tissue engineering, wound dressing or drug delivery systems. ${ }^{3,6-9}$ Although it is a field still in development, Chitin-based materials reports have been increasing since researchers have understood chitin's chemical behaviour and developed promising techniques to obtain these materials. Among them it can be mentioned the 3D chitin scaffolds isolation from marine sponges and biomimetic methods derived from them, chitin hydrogels for biomedical purposes, cross-linked chitin hydrogels for dye sorption and dissolution in ionic liquid for chitin electrospinning. ${ }^{1,6,10-13}$

Polysaccharides and other natural polymers are usually chemically modified in order to tailor their physical and chemical properties, such as mechanical strength, thermal stability or chemical reactivity. ${ }^{14}$ The development of hybrid materials by the incorporation of fillers has been documented as a promising strategy for obtaining improved materials. ${ }^{15-18}$ Some examples are the use of magnetite as nanofiller for carrageenans ${ }^{19}$, cellulose whiskers for alginate-acerola pure ${ }^{20}$, carbon nanotubes and clay for chitosan. ${ }^{21,22}$

Among these approaches special attention has been paid to the use of graphene oxide $(\mathrm{GO})$ in polymer reinforcement and chemical modification. GO can be obtained from graphite by assorted methods among which Hummer's oxidative exfoliation is the most wide spread technique. Graphite consists of stacked nano-sheets of graphene, a single layered 2D hexagonal arrangement of $\mathrm{sp}^{2}$ hybridized carbon atoms. ${ }^{23,24}$ It is considered an alternative to the use of its allotropes, the carbon nanotubes, not only because it can be cheaply obtained from low-cost graphite but also because it has shown higher biocompatibility. ${ }^{25}$ In these matter, Pinto et al reviews the biocompatibility of graphene based materials and conclude that the introduction of hydrophilic groups needs to be considered when the obtaining of biocompatible materials is pursued. ${ }^{26}$ In addition, unlike graphene, GO has in its structure a large number of functional groups such as hydroxyls, epoxides, carbonyls and carboxyls, which endows chemical reactivity. ${ }^{27}$ Chemically exfoliated GO can be obtained with controlled degrees of oxidation by the Modified Hummers' method. ${ }^{28}$ Also, reduced GO can be obtain by tailored chemical and thermal reduction. ${ }^{29}$ These characteristics make GO a promising biopolymer filler for a wide range of applications. ${ }^{30,31}$ Several researchers have reported the use of GO as filler for polysaccharide based materials, such as starch, alginate, agarose, cellulose and chitosan, mainly aiming for mechanical properties reinforcement. There are also studies suggesting the improvement of metal sorption capacity, electrical conductivity and enzyme immobilization performance. ${ }^{32-41}$

This study was motivated by the potentiality of the chitin-GO nanosheets combination. In addition to a mechanical reinforcement, the resulting material would have straightforward applications in the biomedical field and food industry, such as drug delivery and wound dressing, since both are biocompatible and non-toxic. ${ }^{3,6}$ Chitin, by its side, has been used in the food industry for several applications including the formation of biodegradable films, recovery of waste material from food processing discards, immobilisation of enzymes and recovery of proteins for processing purposes.,7 On the other hand, the graphene oxide, less studied in the food application field than chitin has promising properties in the development of food wraps in example due to its oxygen barrier behaviour. ${ }^{42}$ In addition, the chemical groups in GO sheets opens the application fields for chitin, a low reactive polysaccharide. Recently, Gou et al reported that GO enhances mechanical properties of chitin films obtained 
by $\mathrm{NaOH} /$ urea dissolution of the polysaccharide. ${ }^{43}$ Our work shows the properties of a gel-like hybrid material made of chitin, dissolved using methanol/calcium solvent, and reinforced with GO nanosheets. Herein we present the influence of the chitin:GO ratio on the chemical, structural and mechanical properties of the final material. Chitin:GO ratios ranged from higher levels of chitin than GO to ones where GO levels were higher. The hybrids were characterized by Fourier Transform Infrared Spectroscopy, Differential Scanning Calorimetry, Scanning and Transmission Electron Microscopy, ${ }^{13} \mathrm{C}$ solid-state Nuclear Magnetic Resonance and rheological measurements. Water content, water uptake and biodegradability were also studied.

\section{Experimental Section}

\section{Materials}

Natural graphite powder $(<125 \mu \mathrm{m}$ particle size $)$ was purchase from Bitter (UK). Chitin from crab shells (DA: 92\%; $\mathrm{Mr} \approx$ 400,000) was obtained from Fluka (USA). Calcium chloride dihydrate and Methanol were purchased from J.T. Baker (N.J., USA). Water was filtered and deionized with a Milli-Q, Millipore system (Milford, MA, USA). All other reagents were of analytical grade.

\section{Preparation of graphene oxide nanosheets}

GO nanosheets were prepared through the Hummers' method. ${ }^{44}$ In the current synthesis, graphite powder $(2.0 \mathrm{~g})$ and sodium nitrate $(1.0 \mathrm{~g})$ were mixed with sulfuric acid $(46 \mathrm{~mL})$ in an ice bath with sustained agitation for $4 \mathrm{~h}$. Then, potassium permanganate $(6.0 \mathrm{~g})$ was added under stirring. The reaction mixture was kept at $35^{\circ} \mathrm{C}$ for $2 \mathrm{~h}$. Afterwards, $92 \mathrm{~mL}$ of water were added, keeping the solution in the ice bath, and further diluted by the addition of $200 \mathrm{~mL}$ of water after keeping the temperature at $98{ }^{\circ} \mathrm{C}$ for another $2 \mathrm{~h}$. Hydrogen peroxide $(20 \mathrm{~mL})$ was then added to reduce the residual potassium permanganate and stirring continued until the mixture turned brown. Finally, the mixture was centrifuged to obtain the graphite oxide powder. In order to clean out any remnant of salt and acid, the powders were re-dispersed in ultra-pure water and re-centrifuged several times. The powder was finally dried at $80{ }^{\circ} \mathrm{C}$.

The graphite oxide was exfoliated into GO monolayer nanosheets (nGO) by sonication at $35 \mathrm{kHz}$ for $30 \mathrm{~min}$ after dispersion in citrate buffer $(0.4 \mathrm{M} ; \mathrm{pH}$ : 4.2$) .{ }^{45}$ Then the suspension was centrifuged and the pellet was washed with water and then with methanol. The methanol was removed by heating in a stove at $60{ }^{\circ} \mathrm{C}$ and the graphene oxide powder was then stored at room temperature.

Each batch of synthesized GO nanosheet was analysed by Dynamic Light Scattering (DLS, Zetasizer Nano-Zs, Malvern Instruments, Worcestershire, UK). The GO nanosheets hydrodynamic diameter was found to be between $370-400 \mathrm{~nm}$ in all synthesis. The yield of the oxidized graphene can be judged by its carbon-to-oxygen ratio. ${ }^{44}$ The carbon-to-oxygen ratio of the sample was 1.7 .

\section{Preparation of chitin hydrogel and chitin-nGO hybrid materials}

To prepare a transparent calcium solvent, $42.5 \mathrm{~g}$ of calcium chloride dihydrate were suspended in $50 \mathrm{~mL}$ of methanol and refluxed for $30 \mathrm{~min}$ at $82{ }^{\circ} \mathrm{C}$ to a near state of dissolution. One gram of chitin powder was suspended in the calcium solvent and refluxed for $2 \mathrm{~h}$ at $90^{\circ} \mathrm{C}$ with stirring. ${ }^{46}$
Different mass ratios of chitin and nGO were mixed by thorough agitation in order to obtain six types of hybrid materials with different chitin to graphene oxide ratios (Chi:nGO): (24:1), $(12: 1),(3: 1),(1.2: 1),(0.6: 1)$ and $(0.3: 1)$. At the higher ratios Chi is the mayor component and in the lower ratios it is the lowest. Since properties and applications could arise from both components, by studying this range of compositions it was expected that a full coverage of nGO effect on Chi gels would be addressed.

The chitin-nGO mixtures were poured between two glasses spaced by glass slides of known width and then submerged in methanol until they gelled. Finally, the gels were subjected to several water incubations in order to wash out all methanol and $\mathrm{CaCl}_{2}$ residue. Blank chitin gels without nGO were obtained by a similar procedure and named: Chi.

\section{Characterisation of hybrid material}

\section{Water content and Water uptake}

The water content of the matrices was determined with a moisture analyser at constant temperature $\left(80{ }^{\circ} \mathrm{C}\right)(\mathrm{MX}-50, \mathrm{~A} \& \mathrm{D}$ Company, Tokyo, Japan). The water uptake of the composite gels was determined in thin rectangular strips with dimensions of 10 $\mathrm{mm} \times 10 \mathrm{~mm} \times 2 \mathrm{~mm}$. They were dried at $60{ }^{\circ} \mathrm{C}$ until constant weight. Then the matrices were exposed to $94 \%$ relative humidity (RH) for 3 weeks to ensure equilibrium before testing. The water uptake $(\mathrm{Wu})$ of the samples was calculated as follows ${ }^{16}$ :

$\mathrm{Wu}(\%)=\left(\mathrm{W}_{1}-\mathrm{W}_{0}\right) / \mathrm{W}_{0} \times 100 \%$

where $\mathrm{W}_{0}$ and $\mathrm{W}_{1}$ were the weight of the sample before exposure to $94 \% \mathrm{RH}$ and after equilibrium, respectively. An average value of three replicates for each sample was taken.

\section{Microscopical characterisation}

Scanning Electron Microscopy (SEM) images of freeze-dried and gold coated samples were taken using a FEI Quanta 200 microscope. The morphology of the hybrid material was observed using a transmission electron microscope (TEM, Zeiss 109). Prior to TEM observation the samples were fixed with $0.1 \mathrm{M}$ phosphate buffer solutions containing glutaraldehyde $2.5 \%$ and then immersed in $\mathrm{OsO}_{4} 1 \%$. After dehydration in graded series of ethanol, the samples were included in a propylen-oxide/Durcupan epoxy resin. Cuts of ninety nanometres were placed in copper grids. Finally they were stained with Uranyl acetate $(2 \%)$ and Lead citrate $(0.5 \%)$.

\section{Spectroscopic characterisation}

Fourier Transform Infrared (FTIR) transmission spectra were acquired in the range of 4000-450 $\mathrm{cm}^{-1}$ using a FTIR Spectrometer (Nicolet 360). All samples were previously dried for $24 \mathrm{~h}$ at $60{ }^{\circ} \mathrm{C}$ to avoid water related bands interference. Solidstate ${ }^{13} \mathrm{C}$ CP-MAS NMR (cross-polarization-magic angle spinning Nuclear Magnetic Resonance) spectra were performed on a Bruker Avance II-300 spectrometer equipped with a $4 \mathrm{~mm}$ MAS probe. All the NMR experiments were performed at room temperature. The operating frequency for carbons was 75.46 $\mathrm{MHz}$ and the spinning rate was $10 \mathrm{kHz}$. AQ: $41 \mathrm{~ms}, \mathrm{CT}: 1.5 \mathrm{~ms}$, repetition rate $5 \mathrm{~s}$, NS: 1024 .

\section{Differential Scanning Calorimetry}

Differential scanning calorimetry (DSC) measurements were performed on Mettler Toledo 822 equipment (Switzerland) and STARe thermal analysis system version 3.1 software (Mettler 
Toledo AG). DSC plots of freeze-dried samples were obtained at a constant rate of $10{ }^{\circ} \mathrm{C} / \mathrm{min}$, from 0 to $400{ }^{\circ} \mathrm{C}$ and cooling naturally to room temperature under air. The instrument was calibrated using standard compounds (indium and zinc) of defined melting point and heat of melting. All measurements were made in duplicate with $2.0 \pm 0.5 \mathrm{mg}$ of sample mass weighed in an open aluminium pan; an empty pan was used as a reference. The oxidation of the sample was observed as a sharp increase in the energy flow due to the exothermic nature of the oxidation reactions. The onset temperature of the oxidative reaction (OOT) was taken as the intersection of the extrapolated baseline and the tangent line (leading edge) of the exothermal peak.

\section{Mechanical stability}

In order to assess the mechanical stability of the hybrids, discs of $6 \mathrm{~mm}$ diameter and $2 \mathrm{~mm}$ width were subjected to mechanical stirring in liquid media. The discs were immersed in $100 \mathrm{~mL}$ of water and stirred (500 rpm) by using a magnetic bar. The weight loss was determined gravimetrically after 2, 4, and $24 \mathrm{~h}$ incubations at $25^{\circ} \mathrm{C}$. An average value of three replicates for each sample was taken.

\section{Rheological behaviour}

Oscillatory assays were performed using a controlled stress rheometer (Paar Physica MCR 300, Anton Paar GMBH, Germany) equipped with parallel plate (PP30) geometry (30 mmdiameter), with a gap sample between 1 and $2 \mathrm{~mm}$. The slabs were placed between the lower plate of the rheometer and the measuring plate with rough surface (model PP/30), using only as much compression as necessary to provide maximum contact area and minimum slip $(\cong 1 \mathrm{~N})$. Temperature was controlled by an external liquid bath thermostat model Viscotherm VT2 (Anton Paar GmbH, Graz, Austria).

Amplitude sweeps were first performed in order to determine the linear viscoelastic range (LVR). Storage $\left(G^{\prime}\right)$ and loss $\left(G^{\prime \prime}\right)$ shear moduli as well as strain were recorded as a function of stress, at a constant frequency of $1 \mathrm{~Hz}$ and temperature of $20^{\circ} \mathrm{C}$. The constant strain value, at which the following frequency sweeps were performed to obtain the mechanical spectra, was chosen from the originally determined LVR for each sample. Each mechanical spectrum was then recorded at this constant strain value $(\gamma=0.05 \%)$ in the LVR: $G^{\prime}$ and $G^{\prime \prime}$, as well as the tangent of the phase angle $\left(\tan \delta=G^{\prime \prime} / G^{\prime}\right)$ and complex viscosity $\left(\eta^{*}\right)$ were obtained as a function of increasing angular frequency $\left(\omega ; \operatorname{rad~s}^{-1}\right)$, after reaching steady-state condition for each point.

\section{In vitro biodegradability}

In order to assess in vitro biodegradability, discs of $6 \mathrm{~mm}$ diameter and $2 \mathrm{~mm}$ width $(0.2 \mathrm{~g})$ were immersed in $5 \mathrm{~mL}$ of PBS $\left(0.1 \mathrm{~mol} / \mathrm{L} \mathrm{Na}_{2} \mathrm{HPO}_{4} \mathrm{pH} 7.4,0.8 \% \mathrm{NaCl}\right)$ containing lysozyme $(0.06 \%)$ at $37{ }^{\circ} \mathrm{C}$. The lysozyme solution was replaced every two days ${ }^{47}$ After a week of incubation the hybrids were taken out from the lysozyme solution and analysed comparing the degree of rupture of each composite.

\section{Results and Discussion}

\section{Macro and microscopical characterisation}

The material obtained is a gel thick enough to withstand handling. Electronic Supplementary Information 1 (ESI 1)

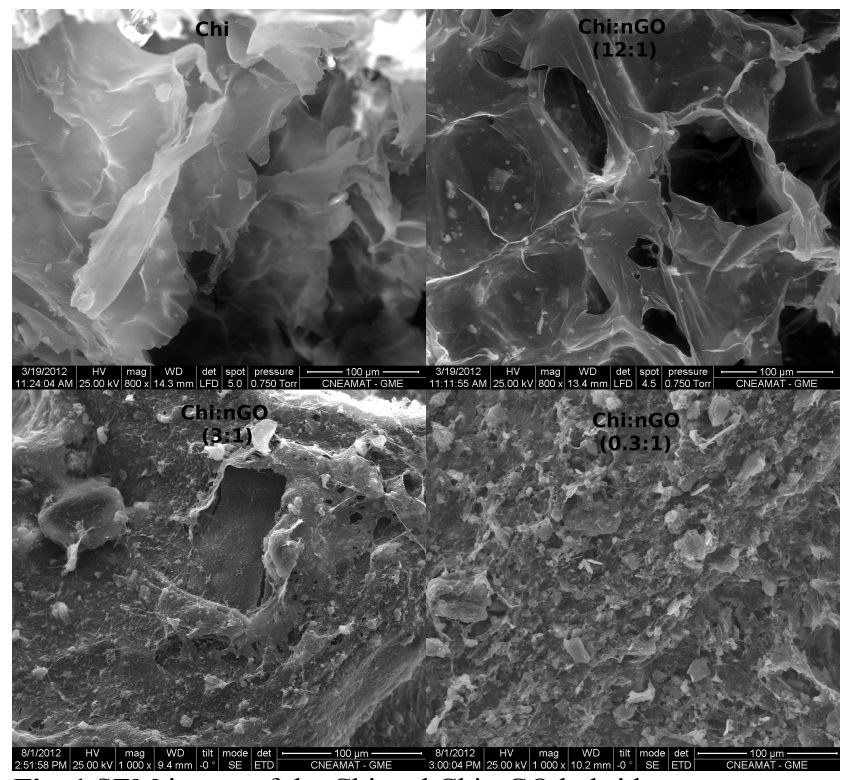

Fig. 1 SEM image of the Chi and Chi:nGO hybrids.

visually shows that the macroscopical stiffness is higher in those gels with higher nGO content. SEM images were used to observe the topography of the materials and how it is altered by the addition of nGO (Fig. 1). Chitin has a smooth and homogeneous surface that is lost as the amount of the inorganic compound increases. ESI 2 shows TEM images of Chi and Chi:nGO hybrids. It can be observed that chitin gel shows a homogeneous network, while those with increasing amounts of nGO show more dense areas with loss of homogeneity. These dark spots are attributed to accumulations of graphene oxide. In the image of the (24:1) hybrid one could observe isolated nanosheets which become more difficult to find at higher percentages; this observation leads to the assumption that the GO is homogeneously dispersed when it is used in low proportion. Therefore, both microscopies demonstrate that when increasing the amount of graphene oxide, it is difficult to achieve a good dispersion of the nanosheets and they tend to self-associate forming dark accumulations visible in TEM images. Other researchers have proposed that GO act as a filler in chitosan-GO composites which can also be the case for these Chi:nGO hybrids. ${ }^{15}$ Guo et al have observed a similar behaviour when the $\mathrm{nGO}$ content was increased in chitin films and also propose that nGO act as a filler in chitin-nGO films. ${ }^{43}$

\section{Water content and Water uptake}

Table 1 presents the water content and water uptake $(\mathrm{Wu})$ results for the different hybrid compositions. The water content variation was statistically analysed by One way ANOVA followed by a Tukey post-test. The water content determination shows that when the amount of nGO increases, the water content of the material decreases significantly when Chi hydrogel is compared with Chi:nGO hybrids $(3: 1)(p<0.05)$ and from $(1.2: 1)$ to lower ratios $(p<0.01)$. Also, between Chi:nGO (3:1) and $(0.3: 1)$ there is a significant drop in the water content $(p<0.01)$, as well as between $(0.6: 1)$ and $(0.3: 1) \quad(p<0.05)$. This probably occurs because the nGO acts as a large nanofiller in the chitin matrix and replaces the pore free water without any swelling effect. On the 
Table 1 Water content $(\mathrm{Wc})$ and Water uptake $(\mathrm{Wu})$ in Chi and Chi:nGO hybrids

\begin{tabular}{|c|c|c|c|c|c|c|c|c|}
\hline \multirow{3}{*}{$\begin{array}{c}\text { Param } \\
\text { eter }\end{array}$} & \multicolumn{7}{|c|}{ Hybrid } & \multirow{3}{*}{ nGO } \\
\hline & \multirow[t]{2}{*}{ Chi } & \multicolumn{6}{|c|}{ Chi:nGO } & \\
\hline & & $24: 1$ & $12: 1$ & $3: 1$ & $1.2: 1$ & $0.6: 1$ & $0.3: 1$ & \\
\hline $\begin{array}{l}\text { Wc } \\
(\%)\end{array}$ & $\begin{array}{c}92.8 \pm \\
0.7\end{array}$ & $\begin{array}{c}91.4 \pm \\
0.3\end{array}$ & $91 \pm 2$ & $88 \pm 2$ & $88 \pm 2$ & $86 \pm 1$ & $\begin{array}{c}81.9 \pm \\
0.4\end{array}$ & n.d. ${ }^{a}$ \\
\hline $\begin{array}{l}\text { Wu } \\
(\%)\end{array}$ & $\begin{array}{c}21.8 \pm \\
0.9\end{array}$ & $23 \pm 2$ & $23 \pm 1$ & $21 \pm 1$ & $22 \pm 1$ & $\begin{array}{c}19.5 \pm \\
0.4\end{array}$ & $\begin{array}{c}18.5 \pm \\
0.7\end{array}$ & $16 \pm 4$ \\
\hline
\end{tabular}

${ }^{\mathrm{a}}$ not determined

other hand, although the $\mathrm{Wu}$ percentage is inversely proportional to the amount of $\mathrm{nGO}$, the drop in $\mathrm{Wu}$ is not steep enough to present significant differences among the hybrids $(p>0.05$, nonparametric test Kruskal-Wallis). This could be explained in terms of two events; first, the large size of GO nanosheets (370-400 $\mathrm{nm})$ prevents the entry of water molecules during the rehydration; and second, after the material drying its size decreases considerably, so it can be accompanied with a rearrangement of the chitin chains around the nGO that hinder the hydration process.

\section{Spectroscopic characterisation}

The FTIR spectra of graphite and graphene oxide are shown in ESI 3. The graphene oxide spectrum shows the increase in the bands corresponding to oxidized groups which confirms the chemical exfoliation of graphite. In the spectrum of nGO, the band at $1240 \mathrm{~cm}^{-1}$ is attributed to the union C-O-C stretching which demonstrates the formation of epoxy groups. The presence of carboxyl and carbonyl functional groups can also be detected at $1400 \mathrm{~cm}^{-1}$ and $1720 \mathrm{~cm}^{-1}$ corresponding to $\mathrm{C}-\mathrm{OH}$ and $\mathrm{C}=\mathrm{O}$ stretchings, respectively. ${ }^{33}$ Fig. 2 presents the spectra of nGO, Chi and Chi:nGO hybrids. The Chi spectrum shows a band at 1660 $1620 \mathrm{~cm}^{-1}$ corresponding to the amide I band; this band involves the doublet at 1655 and $1625 \mathrm{~cm}^{-1}$ (C-O and $\mathrm{C}-\mathrm{N}$ stretching, respectively). Other characteristic bands are amide II band (1540 $\mathrm{cm}^{-1}$, N-H stretching) that is detectable together with amide III band $\left(1390 \mathrm{~cm}^{-1}\right)$ and the glycosidic bond band at $900-1100 \mathrm{~cm}^{-1}$ (C-O-C stretching) ${ }^{48}$ Comparing the spectra, one could see the increment and decrease of the relative intensities of the characteristic bands of each component according to their proportion in the material. For example as nGO increases the intensity of its characteristic bands also increases. In the spectra of the hybrids one could not observe any new band or the disappearing of any band present in Chi or nGO individual spectrum. This probably indicates that the interaction between chitin and nGO would not involve the formation of a new functional group detectable by the FTIR experimental conditions. The FTIR spectra of the other Chi:nGO hybrids are presented in ESI 4.

The ${ }^{13} \mathrm{C}$ CP-MAS NMR of commercial chitin, Chi and Chi:nGO hybrids are shown in Fig. 3. As in FTIR spectra, no additional signals or peak shifts can be observed. On the other hand, the relative intensity of the 73 and $76 \mathrm{ppm}$ signals, corresponding to $\mathrm{C} 3$ and $\mathrm{C} 5$, varies among the spectra. Crab shell chitin, used in this study, appears in the form of $\alpha$-chitin for which typical solid NMR spectrum shows a higher signal for C3 than for C5 in a well-defined doublet. ${ }^{49,50}$ This is also observed in the spectrum of commercial chitin powder. On the contrary, the spectrum of Chi hybrids shows similar intensities for the $\mathrm{C} 3$ and C5 signals. In the spectra of Chi:nGO hybrids there could also be

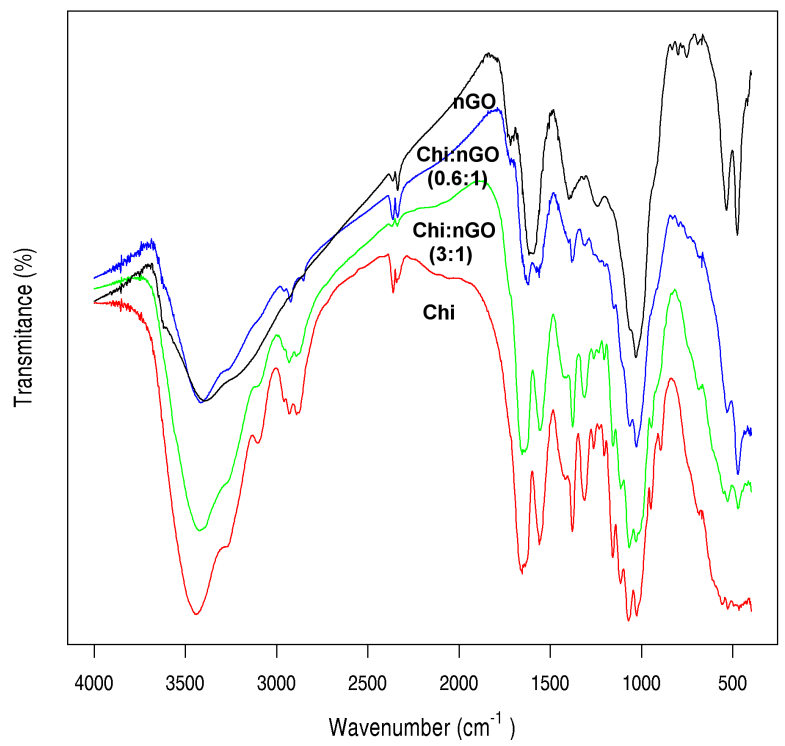

Fig. 2A FTIR spectra of nGO, Chi and Chi:nGO hybrids from $4000-450 \mathrm{~cm}^{-1}$

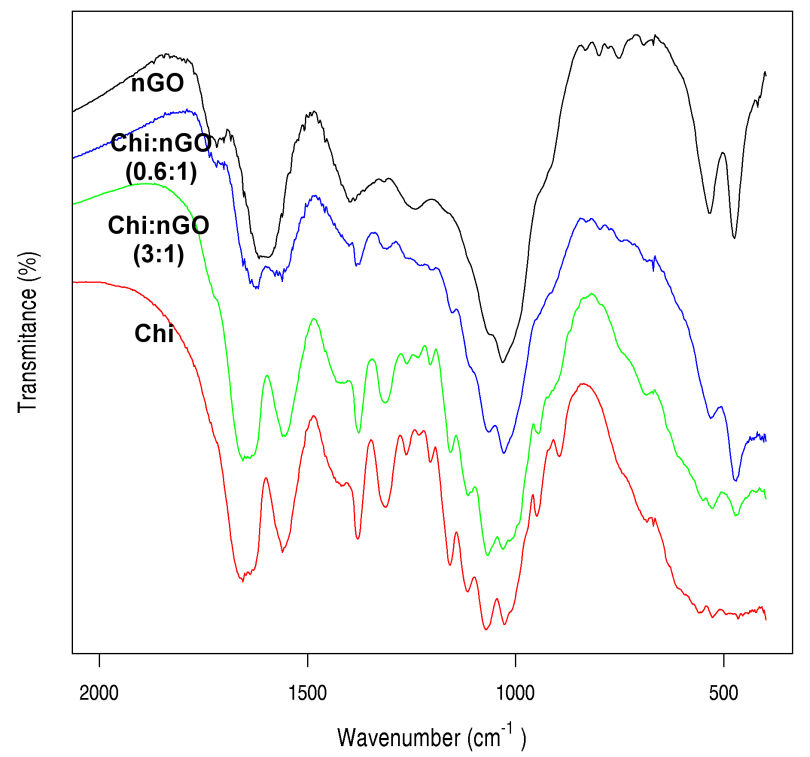

Fig. 2B FTIR spectra of $\mathrm{nGO}$, Chi and Chi:nGO hybrids from $2000-450 \mathrm{~cm}^{-1}$

seen similar intensity of these signals. Also, along with the increment of nGO content, the doublet tends to merge into one signal due to peak broadening. Literature reports that when comparing the ${ }^{13} \mathrm{C}$ CP-MAS NMR spectra of $\alpha, \beta$ and $\gamma$-chitin, the main differences appear in $\mathrm{C} 3$ and $\mathrm{C} 5$ signals when their relative intensities are compared. ${ }^{49,50}$ The variation of these signals was attributed to the possibility of forming different intra and interchain hydrogen bondings by the three polymorphic crystalline structures of chitin. Therefore, comparing FTIR and NMR spectra, it could be proposed that in the process of suspending chitin, the adding of nGO and the subsequent gelling, the $\alpha$-chitin structure is lost and a new structural rearrangement with new hydrogen bondings takes place. In fact, as can be seen in ESI 5, the sole presence of nGO is not the reason of the change in $\mathrm{C} 3$ and C5 signals since the spectrum of a mixture of chitin and nGO powders shows no difference from the commercial chitin powder spectrum. Moreover, the $\mathrm{C} 3$ and $\mathrm{C} 5$ signal broadening observed 


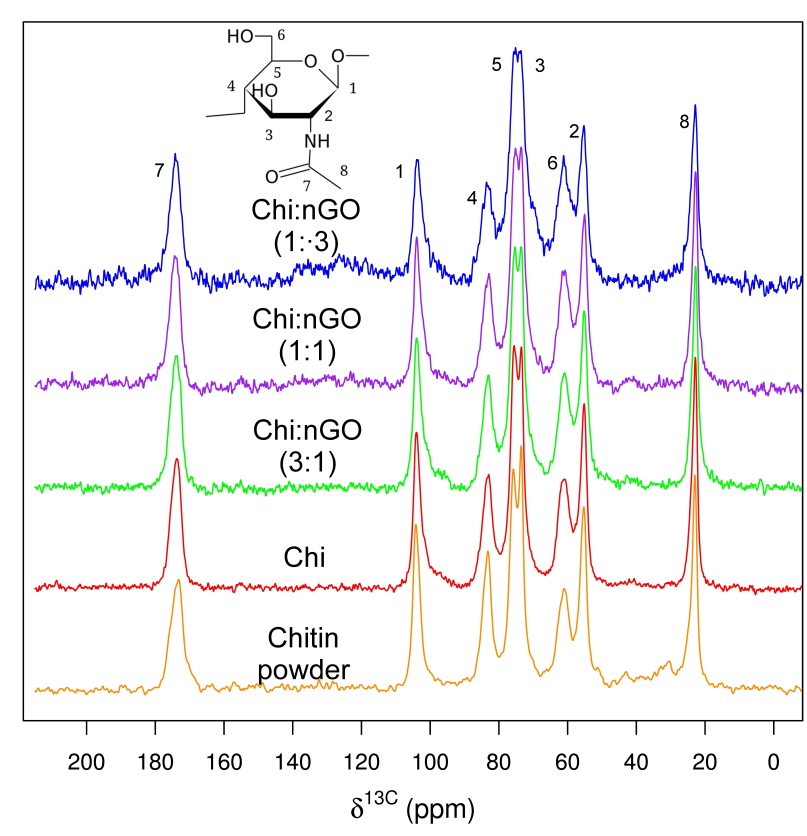

Fig. 3 Solid-state ${ }^{13}$ C-NMR spectra of Chi, Chi:nGO hybrid and commercial chitin powder.

in the Chi:nGO hybrids spectra is not appreciated in the spectrum of the powder mix which demonstrates the influence of the nGO in the hybrids structure. Hence, after the loss of the $\alpha$-chitin structure the nGO oriented rearrangement of chitin chains is probably the reason for the hybrids reinforcement since no chemical bondings or interactions could be demonstrated either by FTIR or by solid NMR spectroscopies. On the other hand, considering the presence of hydrophilic groups in both components it cannot be neglected the possibility of hydrogen bond formations between chitin and nGO. As has been pointed by other researchers on chitin based materials interactions, the lack of any visible changes in the measured spectra indicates that there are no strong interactions among components and weak interactions, such as hydrogen bonds, could be expected. ${ }^{10,51}$

Concerning nGO, it would be expected that being both components of the hybrid in similar proportion, the signals from nGO would appear in the spectra of Chi:nGO hybrids. In none of the spectra are these signals visible. Also, the acquisition of a pure nGO powder spectrum with the same number of scans as the hybrids showed no clear peaks (data not shown). In solid state NMR the homotopic carbons of large and flat molecules, such as nGO, are more likely to have different effective magnetic fields (depending of the carbon position in the molecule) traduced in differences in their chemical shifts. ${ }^{52}$ These would lead to broad bands and lower sensitivity for nGO than for chitin.

\section{Thermal transitions by Differential Scanning Calorimetry}

Fig. 4 shows the DSC thermograms obtained for the Chi, Chi:nGO hybrid materials and the commercial chitin powder. The Chi thermograms showed a broad endothermic peak around $60{ }^{\circ} \mathrm{C}$ and a exothermic event at a temperature higher than $300{ }^{\circ} \mathrm{C}$. The endothermic peak was attributed by other authors to the evaporation of water bound to polar sites of chitin, being changes in this transition associated to different polymorphic crystalline structures $(\alpha, \beta$ and $\gamma)$ of this polysaccharide. ${ }^{50}$ This transition was also observed in both the Chi hydrogel and the commercial chitin powder thermogram at a similar temperature. On the other hand,

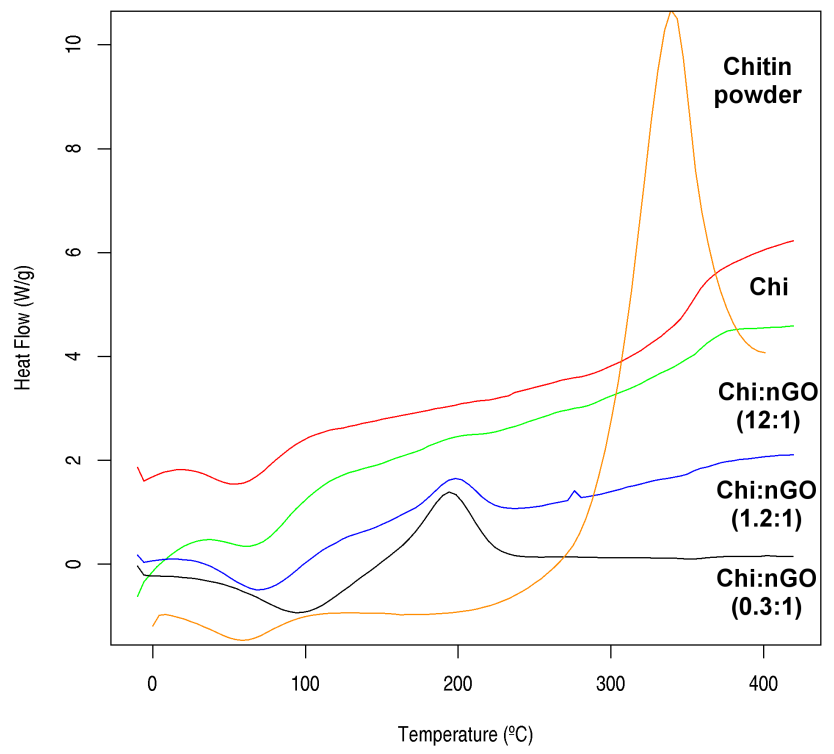

Fig. 4 DSC thermograms of Chi and Chi:nGO hybrids and commercial chitin powder.

the endothermic peak was also observed in the hybrids materials, with a shift towards higher temperatures as the nGO content in the system increases. For the Chi:nGO $(0.3: 1)$ hybrid the endothermic peak occurs at the highest temperature $\left(96^{\circ} \mathrm{C}\right)$, suggesting structural changes in the hybrid material that increase water-solid interactions. This is consistent with the solid state NMR spectra results and supports the premise that the ratio of nGO is involved in the structural arrangement of chitin chains in the hybrids. Near $200{ }^{\circ} \mathrm{C}$ a sharp exothermic event was observed in the nGO containing systems. This peak corresponds to the decomposition of oxygen groups of the $\mathrm{nGO} .{ }^{53}$ As expected, the area of the peak increases with the amount of nGO in the hybrids and was not observed for chitin hydrogel.

The oxidation of the samples was observed as a sharp increase in the energy flow due to the exothermic nature of the oxidation reactions. The onset temperature of the oxidative reaction (OOT) was taken as the intersection of the extrapolated baseline and the tangent line (leading edge) of the exothermal peak. Fig. 4 shows that oxidation process was only evident in those samples that did not undergo decomposition (Chi: absence of exothermic peak at $200^{\circ} \mathrm{C}$ ) or were partially decomposed during heating (Chi:nGO (12:1) and (1.2:1)). In the commercial chitin thermogram this reaction is observed as a rise of the baseline after a sharp exothermic event around $340{ }^{\circ} \mathrm{C}$ which other researchers have attributed to the decomposition of acetyl residues of chitin. ${ }^{54}$ This peak is absent in Chi and Chi:nGO hybrids thermograms. Probably, also this decomposition does not occurs as a sharp and defined event due to the structural arrangement of chitin chains and it is observed as a part of the exothermic increase of energy flow above mentioned.

DSC thermograms were also carried under a protective $\mathrm{N}_{2}$ atmosphere in order to study phase transitions of the materials (ESI 6). In the chitin powder thermogram a sharp endothermic event $(\mathrm{Tm})$ could be observed at $370{ }^{\circ} \mathrm{C}$. For Chi and Chi:nGO hybrids this event was not evident. For Chi thermograms this peak is observed at $310^{\circ} \mathrm{C}$. Again, probably this change in the transition temperature could be due to a new chitin structural arrangement. ${ }^{50}$

\section{Mechanical stability}




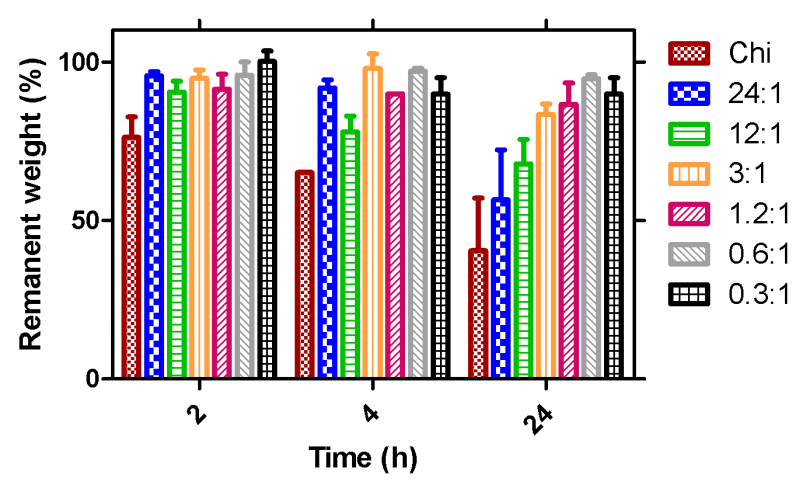

Fig. 5 Remanent weight of the hybrids after incubation with mechanical stirring.

Fig. 5 shows the remaining weight of the hybrids after incubation with mechanical stirring. An important loss of weight is observed in the Chi, Chi:nGO (24:1) and (12:1) hybrids after $24 \mathrm{~h}$ due to a random rupture of the composites. The weight losses of the hybrids with higher nGO content were lower which was probably due to the detachment of small peripherals fragments rather than rupture of the hybrids. The weight loss for ratios from $(3: 1)$ to $(0.3: 1)$ showed to be not significantly different $(p>0.05$, One way ANOVA). Also, the standard deviation of the weight loss of these hybrids showed to be lower, indicating more resistant materials. Therefore, it can be concluded that the cohesion of the material and its mechanical stirring stress resistance is proportional to the amount of nGO up to a limit of Chi:nGO ratio of (12:1) with no significant improvement with higher nGO contents.

\section{Rheological behaviour}

For all composites the $G^{\prime}$ behaviour was independent of frequency in the tested range. Also $G^{\prime}$ was greater than the $G^{\prime \prime}$ for all the cases which implies that the elastic component of the material is dominant over the viscous component, typical of a gellike behaviour. Table 2 presents the comparison of the rheological parameters measured at $10 \mathrm{~Hz}$ and $\gamma=0.05 \%$. It could be seen that all hybrids show similar values of $G^{\prime}$ without a tendency associated with the nGO content. On the contrary, $\tan \delta$ values show to decrease up to $25 \%$ for the higher nGO contents. For gel materials with values of $\tan \delta$ below 1 this drop would be indicative of a more solid-like behaviour. ${ }^{55}$ The complex viscosity of the hybrids decrease almost linearly with the increment of the frequency showing shear thinning behaviour probably due to the structure of the hybrid polymer network (ESI 7).

\section{In vitro biodegradability}

It is well known that chitin is a biodegradable polysaccharide with a glycosidic bond that could be hydrolysed by several enzymes in nature, i.e. lysozyme and chitinase. ${ }^{47}$ ESI 8 shows the hybrid discs before and after lysozyme exposure. After the exposure to lysozyme, it is possible to see an important rupture of all Chi:nGO discs regardless of the proportion of each component. This suggests that the nGO content does not affect or interfere the activity of lysozyme on chitin. In fact, the structural rupture of the hybrid did not allow the weighting of the discs since they disaggregated upon contact. This would also be indicative that the nGO is acting as a nanofiller rather than strongly interacting with chitin chains.

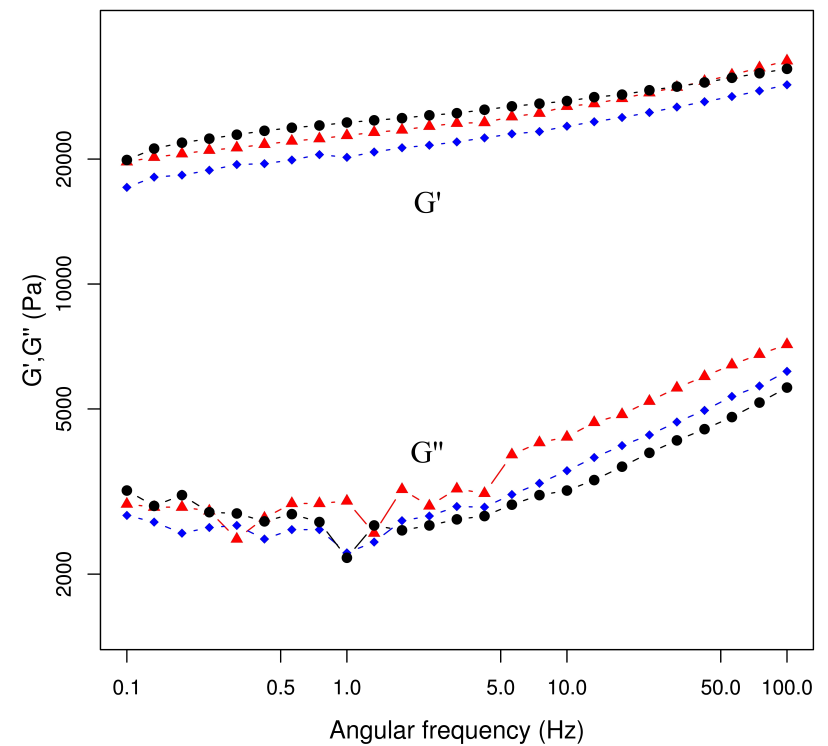

Fig. 6 Storage modulus $\left(G^{\prime}\right)$ and loss modulus $\left(G^{\prime \prime}\right)$ frequency dependence of the hybrids. Symbols: Chi $(\boldsymbol{\Delta})$, Chi:nGO $(1.2: 1)$ $(\diamond)$ and Chi:nGO (0.3:1) (•).

Table 2 Rheological parameters obtained at $\gamma=0.05$ and $10 \mathrm{~Hz}$

\begin{tabular}{cccccccc}
\hline \multirow{2}{*}{$\begin{array}{c}\text { Param } \\
\text { eter }\end{array}$} & \multicolumn{7}{c}{ Hybrid } \\
\cline { 2 - 8 } & Chi & \multicolumn{7}{c}{ Chi:nGO } \\
\cline { 2 - 8 } & & $24: 1$ & $12: 1$ & $3: 1$ & $1.2: 1$ & $0.6: 1$ & $0.3: 1$ \\
\hline$G^{\prime}$ & $26.2 \pm$ & $19 \pm 2$ & $20 \pm 2$ & $14 \pm 2$ & $23.4 \pm$ & $45 \pm 9$ & $27.1 \pm 0.7$ \\
$(\mathrm{kPa})$ & 0.9 & & & & 0.9 & & \\
$G^{\prime \prime}$ & $4.1 \pm$ & $2.9 \pm$ & $3.0 \pm$ & $1.9 \pm 0.3$ & $3.4 \pm$ & $5 \pm 1$ & $3.3 \pm 0.2$ \\
$(\mathrm{kPa})$ & 0.3 & 0.4 & 0.3 & & 0.2 & & \\
$\tan \delta$ & $0.156 \pm$ & $0.151 \pm$ & $0.151 \pm$ & $0.1365 \pm$ & $0.144 \pm$ & $0.115 \pm$ & $0.12 \pm$ \\
& 0.004 & 0.007 & 0.003 & 0.0007 & 0.005 & 0.001 & 0.01 \\
\hline
\end{tabular}

\section{Conclusions}

In this study, hybrid materials made of chitin reinforced with GO nanosheets have been successfully prepared. The structures and mechanical properties of the hybrid materials have proved to be dependent on the chitin to nGO ratio. These hybrid nanocomposites showed that the chitin structural network can be loaded with nGO masses at over three times the amount possible with the polysaccharide. Microscopy images showed that high nGO proportions may turn into nanosheets association. Spectroscopical and DSC analysis showed that the interaction among the components would not involve the formation of new molecular bondings. On the other hand, nGO would act as a filler that induces structural rearrangements in chitin chains causing new hydrogen bondings between chitin chains which lead to changes in mechanical properties of the materials. Mechanical stability and rheological behaviour tests showed that hybrids with higher nGO content were more stable and solid-like materials. Also, the nGO did not interfere with lysozyme activity in chitin chains which indicates that the polymeric matrix of the materials maintain its biodegradability.

\section{Acknowledgements}


J.A.G. is grateful for his doctoral fellowship granted by Universidad de Buenos Aires. M.E.V. is grateful for her doctoral fellowship granted by Consejo Nacional de Investigaciones Científicas y Tecnológicas. This work was supported with grants from Universidad de Buenos Aires (UBACYT 20020100100919 and 20020090300085). Authors would like to thank J. Nesterzak for his technical assistance and N. Dobin-Berstein for language corrections.

\section{Notes and references}

${ }^{a}$ Cátedra de Química Analítica Instrumental, Facultad de Farmacia y Bioquímica, Universidad de Buenos Aires (UBA), IQUIMEFA (UBACONICET), Junín 956, C1113AAD Buenos Aires, Argentina; Tel/fax: +54 11 49648254; E-mail: gcopello@ffyb.uba.ar

${ }^{b}$ Departamento de Industrias, Facultad de Ciencias Exactas y Naturales - Universidad de Buenos Aires y CONICET, Intendente Guiraldes 2160, CP 1428, Buenos Aires, Argentina

$\dagger$ Electronic Supplementary Information (ESI) available: [details of any supplementary information available should be included here]. See DOI: 10.1039/b000000x/

\section{References}

1. M. Wysokowski, V. V. Bazhenov, M. V. Tsurkan, R. Galli, A. L. Stelling, H. Stöcker, S. Kaiser, E. Niederschlag, G. Gärtner, T. Behm, M. Ilan, A. Y. Petrenko, T. Jesionowski, and H. Ehrlich, Int. J. Biol. Macromol., 2013, 62, 94-100.

2. H. Ehrlich, M. Maldonado, K. Spindler, C. Eckert, T. Hanke, R. Born, C. Goebel, P. Simon, S. Heinemann, and H. Worch, J. Exp. Zoolog. B Mol. Dev. Evol., 2007, 308B, 347-356.

3. M. N. . Ravi Kumar, React. Funct. Polym., 2000, 46, 1-27.

4. F. Shahidi, J. K. V. Arachchi, and Y.-J. Jeon, Trends Food Sci. Technol., 1999, 10, 37-51.

5. R. Muzzarelli, Carbohydr. Polym., 1983, 3, 53-75.

6. H. Tamura, T. Furuike, S. V. Nair, and R. Jayakumar, Carbohydr. Polym., 2011, 84, 820-824.

7. F. Wolman, G. Copello, A. Mebert, A. Targovnik, M. Miranda, A. Navarro del Cañizo, L. Díaz, and O. Cascone, Eur. Food Res. Technol., 2010, 231, 181-188.

8. G. J. Copello, A. M. Mebert, M. Raineri, M. P. Pesenti, and L. E. Diaz, J. Hazard. Mater., 2011, 186, 932-939.

9. M. Sharma, C. Mukesh, D. Mondal, and K. Prasad, RSC Adv., 2013, 3, 18149-18155.

10. K. Spinde, M. Kammer, K. Freyer, H. Ehrlich, J. N. Vournakis, and E. Brunner, Chem. Mater., 2011, 23, 2973-2978.

11. H. Ehrlich, P. Simon, M. Motylenko, M. Wysokowski, V. V. Bazhenov, R. Galli, A. L. Stelling, D. Stawski, M. Ilan, H. Stocker, B. Abendroth, R. Born, T. Jesionowski, K. J. Kurzydlowski, and D. C. Meyer, J. Mater. Chem. B, 2013, 1, 5092-5099.

12. M. Wysokowski, M. Motylenko, H. Stocker, V. V. Bazhenov, E. Langer, A. Dobrowolska, K. Czaczyk, R. Galli, A. L. Stelling, T. Behm, L. Klapiszewski, D. Ambrozewicz, M. Nowacka, S. L. Molodtsov, B. Abendroth, D. C. Meyer, K. J. Kurzydlowski, T. Jesionowski, and H. Ehrlich, J. Mater. Chem. B, 2013, 1, 6469 6476.

13. P. S. Barber, C. S. Griggs, J. R. Bonner, and R. D. Rogers, Green Chem., 2013, 15, 601-607.

14. J. Desbrieres and V. Babak, Soft Matter, 2010, 6, 2358-2363.

15.Y. Pan, T. Wu, H. Bao, and L. Li, Carbohydr. Polym., 2011, 83, 1908-1915.

16. M. N. Anglès and A. Dufresne, Macromolecules, 2000, 33, 8344-8353.
17. N. Lin and A. Dufresne, Macromolecules, 2013, 46, 55705583.

18. H. Feng, Y. Li, and J. Li, RSC Adv., 2012, 2, 6988-6993.

19. A. L. Daniel-da-Silva, J. Moreira, R. Neto, A. C. Estrada, A. M. Gil, and T. Trindade, Carbohydr. Polym., 2012, 87, 328-335.

20. H. M. C. Azeredo, K. W. E. Miranda, H. L. Ribeiro, M. F. Rosa, and D. M. Nascimento, J. Food Eng., 2012, 113, 505-510.

21.. C. Tang, L. Xiang, J. Su, K. Wang, C. Yang, Q. Zhang, and Q. Fu, J. Phys. Chem. B, 2008, 112, 3876-3881.

22. S.-F. Wang, L. Shen, W.-D. Zhang, and Y.-J. Tong, Biomacromolecules, 2005, 6, 3067-3072.

23. V. Singh, D. Joung, L. Zhai, S. Das, S. I. Khondaker, and S. Seal, Prog. Mater. Sci., 2011, 56, 1178-1271.

24. A. Y. W. Sham and S. M. Notley, Soft Matter, 2013, 9, 66456653.

25. K.-H. Liao, Y.-S. Lin, C. W. Macosko, and C. L. Haynes, ACS Appl. Mater. Interfaces, 2011, 3, 2607-2615.

26. A. M. Pinto, I. C. Gonçalves, and F. D. Magalhães, Colloids Surf. B Biointerfaces, 2013, 111, 188-202.

27. X. Yang, C. Chen, J. Li, G. Zhao, X. Ren, and X. Wang, RSC Adv., 2012, 2, 8821-8826.

28. K. Krishnamoorthy, M. Veerapandian, K. Yun, and S.-J. Kim, Carbon, 2013, 53, 38-49.

29. C. Botas, P. Alvarez, C. Blanco, M. D. Gutierrez, P. Ares, R. Zamani, J. Arbiol, J. R. Morante, and R. Menendez, RSC Adv., 2012, 2, 9643-9650.

30. C. Wan, M. Frydrych, and B. Chen, Soft Matter, 2011, 7, 6159-6166.

31. A. He, B. Lei, C. (Sage) Cheng, S. Li, L. Ma, S. Sun, and C. Zhao, RSC Adv., 2013, 3, 22120-22129.

32. Y. He, N. Zhang, Q. Gong, H. Qiu, W. Wang, Y. Liu, and J. Gao, Carbohydr. Polym., 2012, 88, 1100-1108.

33. R. Li, C. Liu, and J. Ma, Carbohydr. Polym., 2011, 84, 631637.

34. N. Zhang, H. Qiu, Y. Si, W. Wang, and J. Gao, Carbon, 2011, 49, 827-837.

35. J.-D. Qiu, J. Huang, and R.-P. Liang, Sens. Actuators B Chem., 2011, 160, 287-294.

36. A. A. Alhwaige, T. Agag, H. Ishida, and S. Qutubuddin, RSC Adv., 2013, 3, 16011-16020.

37. C.-J. Cai, M.-W. Xu, S.-J. Bao, C. Lei, and D.-Z. Jia, RSC Adv., 2012, 2, 8172-8178.

38. Y. Wang, P. Zhang, C. F. Liu, and C. Z. Huang, RSC $A d v$, 2013, 3, 9240-9246.

39. Y. Feng, X. Zhang, Y. Shen, K. Yoshino, and W. Feng, Carbohydr. Polym., 2012, 87, 644-649.

40. D. Han, L. Yan, W. Chen, W. Li, and P. R. Bangal, Carbohydr. Polym., 2011, 83, 966-972.

41. D. Han, L. Yan, W. Chen, and W. Li, Carbohydr. Polym., 2011, 83, 653-658.

42. H. M. Kim, J. K. Lee, and H. S. Lee, Thin Solid Films, 2011, 519, 7766-7771.

43. Y. Guo, B. Duan, J. Zhou, and P. Zhu, Cellulose, 2014, 1-11.

44. W. S. Hummers and R. E. Offeman, J. Am. Chem. Soc., 1958, 80, 1339.

45. Q. Bao, D. Zhang, and P. Qi, J. Colloid Interface Sci., 2011, 360, 463-470.

46. H. Tamura, H. Nagahama, and S. Tokura, Cellulose, 2006, 13, 357-364.

47. Y. M. Yang, W. Hu, X. D. Wang, and X. S. Gu, J. Mater. Sci. Mater. Med., 2007, 18, 2117-2121.

48. Y. Saito, J.-L. Putaux, T. Okano, F. Gaill, and H. Chanzy, Macromolecules, 1997, 30, 3867-3873.

49. S. F. Tanner, H. Chanzy, M. Vincendon, J. C. Roux, and F. Gaill, Macromolecules, 1990, 23, 3576-3583. 
50. M.-K. Jang, B.-G. Kong, Y.-I. Jeong, C. H. Lee, and J.-W. Nah, J. Polym. Sci. Part Polym. Chem., 2004, 42, 3423-3432.

51. D. Schleuter, A. Günther, S. Paasch, H. Ehrlich, Z. Kljajić, T. Hanke, G. Bernhard, and E. Brunner, Carbohydr. Polym., 2013, 92, 712-718.

52. S. Stankovich, D. A. Dikin, R. D. Piner, K. A. Kohlhaas, A. Kleinhammes, Y. Jia, Y. Wu, S. T. Nguyen, and R. S. Ruoff, Carbon, 2007, 45, 1558-1565.

53. M. J. McAllister, J.-L. Li, D. H. Adamson, H. C. Schniepp, A. A. Abdala, J. Liu, M. Herrera-Alonso, D. L. Milius, R. Car, R. K. Prud'homme, and I. A. Aksay, Chem. Mater, 2007, 19, 43964404.

54. L. S. Guinesi and É. T. G. Cavalheiro, Thermochim. Acta, 2006, 444, 128-133.

55. M. V. Tzoumaki, T. Moschakis, and C. G. Biliaderis, Carbohydr. Polym., 2013, 95, 324-331. 


\section{Table of contents}

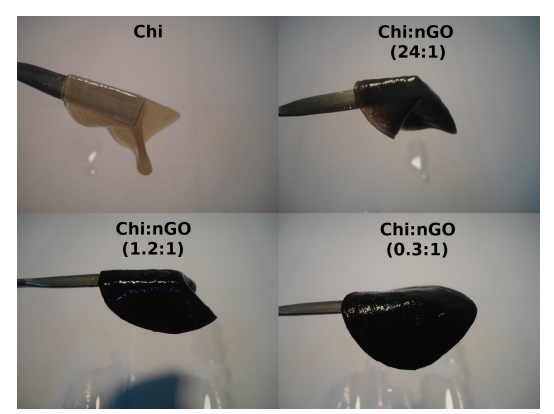

A novel hybrid material of Chitin:nGO was obtained. Component ratios ranged from (1:0.04) to (0.3:1). nGO reinforces the material by rearrangement of chitin chains improving its chemical and mechanical properties. 